Meta

Journal des traducteurs

Translators' Journal

\title{
« ... ce que je ne doute » : traduire à la Renaissance
}

\section{Véronique Duché-Gavet}

Volume 61, numéro 1, mai 2016

Des zones d'incertitudes en traduction

URI : https://id.erudit.org/iderudit/1036983ar

DOI : https://doi.org/10.7202/1036983ar

Aller au sommaire du numéro

\section{Éditeur(s)}

Les Presses de l’Université de Montréal

ISSN

0026-0452 (imprimé)

1492-1421 (numérique)

Découvrir la revue

Citer cet article

Duché-Gavet, V. (2016). « ... ce que je ne doute » : traduire à la Renaissance.

Meta, 61(1), 60-77. https://doi.org/10.7202/1036983ar

\section{Résumé de l'article}

L'exemple du passé peut sans nul doute aider à limiter les incertitudes qui compliquent l'acte de traduire. La Renaissance offre alors un terrain particulièrement propice à la comparaison. En effet, tandis que l'humanisme trouve son expression dans un afflux de traductions, tant de textes des Anciens que de textes contemporains, nombreuses sont les zones d'incertitude qui jalonnent le parcours des traducteurs de la Renaissance. Qu'elles soient d'ordre matériel, textuel, pragmatique ou linguistique, ces incertitudes se voient amplifiées par des considérations culturelles ou politiques, voire commerciales. L'incertitude gouverne enfin le genre même de la traduction, si proche de l'imitation, de la paraphrase ou de l'adaptation. Le présent article examine la posture des traducteurs dans la France du xvi ${ }^{\mathrm{e}}$ siècle en analysant leurs discours sur la traduction tout comme leurs productions, et dégage les tactiques et stratégies déployées par ces traducteurs dans leur combat contre l'incertitude. 


\title{
«... ce que je ne doute»: traduire à la Renaissance
}

\author{
VÉRONIQUE DUCHÉ-GAVET \\ The University of Melbourne, Melbourne, Australie \\ veronique.duche@unimelb.edu.au
}

\begin{abstract}
RÉSUMÉ
L'exemple du passé peut sans nul doute aider à limiter les incertitudes qui compliquent l'acte de traduire. La Renaissance offre alors un terrain particulièrement propice à la comparaison. En effet, tandis que l'humanisme trouve son expression dans un afflux de traductions, tant de textes des Anciens que de textes contemporains, nombreuses sont les zones d'incertitude qui jalonnent le parcours des traducteurs de la Renaissance. Qu'elles soient d'ordre matériel, textuel, pragmatique ou linguistique, ces incertitudes se voient amplifiées par des considérations culturelles ou politiques, voire commerciales. L'incertitude gouverne enfin le genre même de la traduction, si proche de l'imitation, de la paraphrase ou de l'adaptation. Le présent article examine la posture des traducteurs dans la France du $\mathrm{xvI}^{\mathrm{e}}$ siècle en analysant leurs discours sur la traduction tout comme leurs productions, et dégage les tactiques et stratégies déployées par ces traducteurs dans leur combat contre l'incertitude.
\end{abstract}

\section{ABSTRACT}

The example of the past can undoubtedly help limiting the uncertainty, which makes translating so difficult. The Renaissance offers a particularly interesting field for comparison. As humanism shows with its increase in translations, from ancient to contemporary texts, many are the hurdles that are scattered throughout the path of Renaissance translators. These difficulties, either material (dealing with the quality of the medium), textual (reliability of the source-text), pragmatical (absence of tools such as dictionaries, glossaries or grammars), or linguistic (bad knowledge of the source-language, absence of flexibility of the target-language), get accentuated by cultural, political or even commercial considerations. Finally ambiguity can be found in the very genre of translation, so close to imitation, paraphrase or adaptation. This article examines the position of French $16^{\text {th }}$ century translators; it analyses their discourse on translation as well as their own translations and identifies the strategies they used in their fight against uncertainty.

\section{MOTS CLÉS/KEYWORDS}

Renaissance, humanisme, incertitude, paratexte, outils de traduction Renaissance, humanism, uncertainty, paratext, translation tools

Si tu y vois autre faute legere (ce que je ne doute) je te prie te souvenir que c'est chose bien difficile de ne s'esgarer en un chemin par où personne n'a encore passé.

(Horace $1579, \mathrm{f}^{\mathrm{o}}$ e iij $\left.\mathrm{v}^{\mathrm{0}}\right)^{1}$

\section{Introduction}

Si nous assistons aujourd'hui à la professionnalisation de la traduction, à la mobilisation des traducteurs en faveur d'une communication accrue et à une prise de conscience aiguë de l'existence de ces zones d'incertitude qui compliquent l'acte de 
traduire, il paraît légitime de se demander dans quelle mesure l'exemple du passé pourrait aider à faire reculer le flou, l'ambigu et le doute. En effet, la Renaissance, qui se distingue des autres siècles en matière de traduction, comme l'ont montré les travaux d'Antoine Berman par exemple $(2012)^{2}$, semble offrir un terrain propice à la comparaison. Depuis les travaux pionniers de Paul Chavy (1988) et de Henri Van Hoof (1991), l'intérêt pour l'histoire de la traduction s'est renouvelé, et de belles études ont été consacrées aux traducteurs (Worth 1988; Delisle et Woodworth 1995) comme aux traductrices (Delisle 2002; Belle 2012). Mais si les problèmes déontologiques rencontrés par les acteurs de la traduction ont parfois fait l'objet d'études générales (voir par exemple Pym 1997), rares sont les études spécifiquement consacrées aux difficultés rencontrées par les traducteurs à l'époque de la Renaissance. Il s'agira ici d'examiner la posture des traducteurs dans la France du $\mathrm{XvI}^{\mathrm{e}}$ siècle, d'analyser leurs discours sur la traduction tout comme leurs versions elles-mêmes. On pourra ainsi dégager les stratégies déployées par ces traducteurs dans leur combat contre l'incertitude, et voir dans quelle mesure ces tactiques sont toujours employées.

\section{Les zones d'incertitude}

L'humanisme qui gagne la France à l'automne du Moyen Âge trouve son expression dans un afflux de traductions, qui diffusent en français les textes des Anciens tout comme les ouvrages contemporains. Toutefois, nombreuses sont les zones d'incertitude qui jalonnent le parcours des traducteurs de la Renaissance. Qu'elles soient d'ordre matériel ou textuel, pragmatique ou linguistique, ces incertitudes se voient amplifiées par des considérations culturelles ou politiques, voire commerciales.

\subsection{Domaine matériel et textuel}

Les premières incertitudes sont tout d'abord d'ordre matériel; sont en jeu la qualité du support ainsi que la fiabilité du texte source. En effet, si la seconde moitié du $\mathbf{X v}^{\mathrm{e}}$ siècle signe le passage du manuscrit à l'imprimé, cette transition n'est pas sans poser de problème, notamment d'ordre typographique. Peu nombreux par exemple sont les imprimeurs disposant de fontes pour les langues rares - l'Anversois Thierry Martens a été le premier à introduire des caractères grecs et hébreux (Adam et Vanaugaerden 2009). Les traductions offrant des passages bilingues présentent souvent une gageure pour les imprimeurs. Philippe Renouard (1969) s'est intéressé au matériel typographique de l'humaniste d'origine belge Josse Bade et rapporte les démêlés de celui-ci avec Érasme pour ce qui est de l'usage des caractères grecs. En effet, la correspondance d'Érasme, entre 1512 et 1518, «fait plusieurs fois allusion à l'insuffisance de la typographie grecque de Josse Bade. [...] Érasme s'inquiète de savoir si Bade aura assez de caractères grecs pour les Adagia, puisque l'ouvrage est pour moitié en grec» (Renouard 1969: 17). On pourra citer en exemple le célèbre Adage 1 "Amicorum communia omnia (Toutes les choses doivent être communes aux amis)» pour lequel Érasme cite dans leur langue «Platon au livre V des Lois» et Théophraste. Un mauvais choix de matériel typographique peut en outre desservir une traduction et en brouiller la lecture: l'utilisation par Pierre de Vingle d'une fonte gothique en voie d'obsolescence, alors que s'imposent les caractères romains, pour 
son édition de la Bible d'Olivétan (1535), a très vraisemblablement contribué au mauvais accueil reçu par cette traduction.

Nombreux en outre sont les traducteurs qui, à la Renaissance, déplorent la corruption du texte de base qu'ils utilisent pour leur travail. Ainsi de Claude Gruget traduisant Les diverses leçons de Pierre Messie (Mexia 1526). L'épître liminaire adressée à «Monseigneur François de Raconis Conseiller du Roy» témoigne des difficultés rencontrées par le traducteur. S’il s'appuie à la fois sur le texte original espagnol et sur une traduction italienne pour composer la version qui lui a été commandée, malheureusement ses deux textes sources ne sont pas de qualité suffisante:

me sentant quelque peu de loisir, j'en [la traduction] entrepris la charge, avec telle affection, que ny l'impression mauvaise de l'une, et l'autre langue Espagnole, et Italienne, ny la dépravation du texte en plusieurs endroits imparfait et corrompu, ne m'ont peu destourner du desir que j'avois de vous complaire en cela... (Mexia 1526: fo ã ij vo)

Une possible stratégie pour lutter contre cette incertitude matérielle est décrite par Annibal Cruceio dans sa traduction latine du roman d'Achille Statius Clitophon et Leucippe. Dans son épître à "Pierre François Palavicin», traduite par François de Belleforest dans sa version française du roman, Annibal Cruceio dénonce:

la corruption de l'exemplaire grec, pour laquelle [lui] a fallu quelque fois deviner, et remettre de [lui] mesmes aucunes choses en leur entier à ce que par effaceures et croix le livre ne fust du tout difforme et enlaidy. Ce qu ['il a] fait en aucuns lieux [...]. (Achille Statius 1575: fo ã ij vo - ã iij $\mathrm{r}^{\circ}$ )

Annibal Cruceio donne pour exemple la «description de la fleuste, ou flajolet» qu'il avait omise lors de la première édition de sa traduction, parce que «le passage qui en parloit estoit imparfait et corrompu » : il l'a alors «composée selon [s]a fantaisie et jugement» (Achille Statius 1575: fo ã iij $\mathrm{r}^{\circ}$ ).

Claude Gruget, pour sa traduction de Pierre Messie mentionnée plus haut, agit de même et «donne quelque peu du sien », se faisant toutefois aider dans sa tâche par deux spécialistes:

[...] j'ay esclaircy des choses obscures, et corrigé plusieurs textes alleguez faux, et s'il est permis de le confesser, j'ai donné quelque peu du mien en des passages, qui selon mon jugement, le requeroient. Peu de mien, dis-je, pour ce que deux de mes amis m'y ont favorisé, l'un desquels est le seul de mes cousins, portant mon nom, et l'autre le seigneur Jean Pierre de $[\mathrm{M}]$ esmes, qui pour les mathematiques, et poincts concernans l'Astrologie (esquelles sciences il faict profession) m'a grandement secouru. (Mexia 1526: fo ã ij $v^{0}-\tilde{a}$ iij $r^{\circ}$ )

Corrompu en amont, le texte peut également l'être en aval. On constate en effet des erreurs commises lors de l'impression, qui nuisent à la qualité du travail et rendent la traduction incertaine. Ainsi de Jacques Mondot déplorant dans son épître «Au lecteur» les «fautes advenues à l'impression» des Cinq livres des Odes de Q. Horace Flacce:

[...] j’ay tousjours la plume en main pour le mettre en meilleur ordre aux suivantes editions, je sçay bien que mon livre rougira souvent des fautes advenues à l'impression, lesquelles je t'ay mis à la fin pour ma charge, au moins les plus notables. Car les yeux d'Aigle ny de Linx n'y verroient encore assez. (Horace 1579: $\mathrm{f}^{\mathrm{o}}$ e iij $\mathrm{v}^{\mathrm{o}}$ ) 
Les deux pages finales du volume corrigent les coquilles (ment pour meut; sonneurs pour honneurs, etc.) et signalent les vers superfétatoires ou manquants. Mondot termine toutefois sur un constat désabusé: «Je ne doute point (Amy lecteur) que tu ne trouve (sic) plusieurs autres fautes tant au Latin qu'au François [...]» (Horace 1579: fo $5 \mathrm{v}^{\circ}$ ).

Texte corrompu, pages manquantes: les traducteurs tentent toutefois de remédier à ces incertitudes matérielles. Une stratégie s'impose au fil du temps : la retraduction. L'histoire éditoriale de l'Elegia di madonna Fiammetta en fournit une bonne illustration. Dans son étude du roman de Boccace, Serge Stolf distingue deux moments: «le premier, couvrant la décennie 1531-1541 [...] voit paraître cinq éditions; le second, marqué par la publication, en 1585, de la traduction de Gabriel Chappuys, et se prolongeant par trois rééditions de cette même version $(1609,1619,1622)$ (2008). La première traduction, anonyme, date de 1531, et a été publiée à Paris pour Jean Longis ${ }^{3}$. Cependant seuls les six premiers chapitres du texte original ont été traduits. Stolf (2008: 180), à la suite de Hauvette (1909), «relevant les nombreux contresens et bévues accumulés», montre que le traducteur "travaillait sur une édition italienne de mauvaise qualité», "un texte italien corrompu difficile à interpréter». Selon le critique, «la traduction française se caractérise par deux autres aspects notables: les interpolations, et les coupures». Stolf en déduit « une compréhension approximative du texte italien, passablement malmené». Une seconde traduction, intégrale cette fois-ci, voit le jour en 1585, sous la plume de Gabriel Chappuys, et permet de lever les incertitudes. La Fiammette amoureuse de M. Iean Boccace gentil-homme florentin est donnée en édition bilingue, et la traduction, présentée en regard du texte italien, est juxtalinéaire. Le texte de l'Elegia de Boccace est en conformité avec celui qu'établissent les éditions contemporaines. Selon Stolf (2008: 188), «la qualité globale de la traduction est bonne» et c'est en 2003 seulement que le texte a été retraduit ${ }^{4}$. Un texte source de meilleure qualité a donc permis d'améliorer la version en français.

De la même façon, après avoir mis la main, au Vatican, sur un manuscrit dont le texte différait légèrement de celui qu'il avait utilisé en 1547 pour sa traduction de l'Histoire athiopique, Jacques Amyot publie en 1559 une nouvelle version du roman d'Héliodore d'Emèse, "Traduite de Grec en François, et de nouveau reveüe et corrigée sur un ancien exemplaire escript à la main, par le translateur», qui lui offre également l'occasion de "declar[er] au vray qui en a esté le premier autheur», Héliodore. Lors de la première édition de sa traduction, Amyot signalait les difficultés qu'il avait rencontrées, et la méthode qu'il avait adoptée:

[...] si d'avanture mon jugement m'a trompé en restituant par conjecture aucuns lieux corrompuz, et vicieusement imprimez, les equitables lecteurs m'en devront plustost excuser: tant pource que je n'ay peu recouvrer diversité d'exemplaires, pour les conferer, que pourautant que j'ay esté le premier qui l'ay traduit, sans estre du labeur d'aucun precedant aydé. D’une chose me puis-je bien vanter, que je ne pense y avoir rien omis, ny ajousté, ainsi comme les lecteurs le pourront trouver, s'il leur plaist prendre la peine de le conferer. (Héliodore 1547: ff. Aiij vº-Aiv rº)

La comparaison avec le manuscrit trouvé à la Vaticane lui permet de vérifier la qualité de sa première traduction:

[...] en conferant l'examplaire imprimé avec ce vieil escrit à la main, j’ay trouvé que ma conjecture ne m'avoit point trompé en restituant plusieurs endroitz vicieusement imprimez, que j'avois corrigé par jugement seulement: mais aussi ay je trouvé en plusieurs 
endroitz des lignes entieres omises en l'impression Grecque, lesquelles je cottoys en la marge de mon livre imprimé. (Héliodore 1559: Proesme du translateur, $\mathrm{f}^{\circ}$ iij $\mathrm{v}^{\circ}$ )

Ainsi Amyot se félicite-t-il d'avoir «reveu[...], rempli[...] et emendé[...]» sa traduction, et estime-t-il que:

[...] qui aura l'original Grec entier et correct, s'il luy plaist prendre la peine de conferer [s]a traduction avec le Grec, trouvera que [il] n’y a[...] à [s]on advis rien adjousté ny omis. (Héliodore 1559: Proesme du translateur, $\mathrm{f}^{\mathrm{o}}$ iij $\mathrm{v}^{\circ}$ )

Sébastien Castellion agit avec le même zèle lorsqu'il traduit en latin les Oracula Sibylina, ce recueil de textes poétiques composés en grec. Castellion offre une première traduction en 1546, que publie le célèbre imprimeur bâlois Jean Oporin ${ }^{5}$. JeanMichel Roessli (2013: 228-231) a mis en évidence la méthode d'un humaniste éditant avec passion les textes antiques et toujours prêt à émender son texte. Ainsi Castellion suit les recommandations que lui adresse le philologue Marc Antoine Antimaque (Marcantonio Antimaco), qui était en possession d'un manuscrit ancien ("vetus exemplar») présentant plusieurs variantes par rapport au codex Betuleius utilisé pour la première version. Une édition bilingue voit ensuite le jour en 1555, chez le même imprimeur Oporin, corrigée et enrichie de substantielles annotations par Castellion. La longue épître liminaire qu'il adresse à Antoine Morelet du Museau montre toutefois que l'érudit est à la recherche d'un troisième manuscrit qu'il souhaite collationner pour améliorer encore sa version.

On saluera ainsi le travail de fond accompli par certains traducteurs, véritables philologues soucieux de la qualité de leur texte source tout comme de l'excellence de leur travail, et parmi lesquels on compte Jean de Maumont. Traducteur des Euvres de sainct Justin Philosophe et martyr, mises de Grec en langage François (Justin 1559), Maumont revendique toute responsabilité du texte qu'il offre au lecteur, arguant de son «ignorance». Cependant, il s'en prend aussi à la mauvaise qualité de son texte source:

Et au reste, des defauts, Lecteur, que tu trouveras en la presente translation, je ne veux pas nier que mon ignorance n'en soit la principale, mais j'ose bien dire qu'elle n'en est pas la seule cause: car l'exemplaire Grec corrompu en plusieurs lieux, y a certes bien aidé. (Justin 1559: fob6 vo

Il fournit un travail minutieux de comparaison, comme l'atteste l'inscription sur la page de titre de son ouvrage:

C’est la seconde edition, reveuë et corrigee par le translateur, et conferee sur l'exemplaire Grec, et sur les traductions Latines, qui en ont esté depuis faites tant en France qu'en Allemagne, avec les additions et corrections necessaires, mises à la fin du livre en un traicté à part, auquel traicté, tous les lieus obscurs et difficiles sont aussi selon les trois traductions declarez et esclarcis, avec un prologue adjousté par le mesme translateur, au tres Chrestien Roy de France Henry, second de ce nom, pour l'edition et translation des livres Grecs, qui sont en sa grande et Royale bibliotheque. (Justin 1559: page de titre)

Le sérieux de la démarche philologique de Maumont transparaît dans l'abondant matériel paratextuel qui accompagne sa traduction, comme en témoigne la postface adressée au lecteur (Justin 1559: ff 318-319) ou encore l'"Advertissement au lecteur",

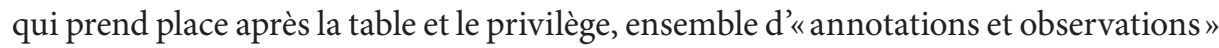


(Justin 1559: NP, fo 338) qui commentent les différences entre les trois traductions qu'il a compilées pour son ouvrage.

On pourra encore saluer la démarche d'Antoine Du Pinet, soucieux de corriger les imperfections de son exemplaire de base pour sa traduction de l'Histoire du monde de Pline (1562):

Quant à moy l'ordre que j'ay tenu à corriger les fautes que j'ay trouvees, a esté par la conference de plusieurs Exemplaires, et par avoir recours aux originaux dont Pline auroit transompté son dire: comme à Aristote, Theophraste, Cornelius Tacitus, Columella, Varro, Cato, Scribonius largus, Dioscorides, Vitruvius, et plusieurs autres: au moyen de quoy, avec l'ayde aussi de plusieurs modernes, j'ay restitué plus de douze centz passages corrompuz. (Pline 1562: «Au Lecteur debonnaire», $\mathrm{f}^{\circ} \mathrm{i}$ )

On a ainsi un aperçu du combat des traducteurs de la Renaissance contre les incertitudes matérielles ou textuelles qu'ils rencontrent, à une époque où l'imprimerie vient bouleverser la circulation des textes et de leurs traductions, et de leurs stratégies pour assurer la qualité de leur travail. Mais les incertitudes sont également d'un autre ordre.

\subsection{Domaine pragmatique et linguistique}

En effet, l'absence d'outils tels que dictionnaires, lexiques ou grammaires, la méconnaissance de la langue source tout comme l'absence de ductilité de la langue cible viennent compliquer la tâche du traducteur à l'aube de la Renaissance.

Certes, pour la langue latine un bon nombre de compilations médiévales circulent encore sous forme manuscrite, comme celle de Papias ( $\mathrm{xI}^{\mathrm{e}}$ siècle), Hugutio (XII ${ }^{\mathrm{e}}$ et XIII ${ }^{\mathrm{e}}$ siècles), la Summa quæe vocatur Catholicon de Giovanni de Balbi (Jean de Gênes), somme achevée en 1286, ou encore le dictionnaire latin-français établi par le religieux cartusien Firmin Le Ver (1440), ouvrages susceptibles de venir en aide au traducteur. On citera aussi le Vocabularius nebrissensis édité pour la première fois à Lyon par Simon Vincent en 1511 et qui se fonde sur le dictionnaire latin-espagnol d'Antonio Nebrija. Mais c'est à Robert Estienne que l'on doit des ouvrages qui font date dans l'histoire de la lexicographie française, le Dictionarium seu Latince linguce Thesaurus (1531), le Dictionarium latinogallicum (1538) et le Dictionnaire françaislatin (1539). Selon Martine Furno, ces ouvrages « ne sont réellement des dictionnaires de traduction, puisqu'ils se limitent à donner une explication vernaculaire, parfois fort large, d'une difficulté du latin» (Furno 1999-2000: 24). Ils signalent néanmoins un jalon important dans l'histoire de la traduction.

Il paraît douteux en revanche que les traducteurs aient fait usage des petits lexiques pratiques, consacrés au vocabulaire de la vie quotidienne, qui circulaient dans des formats portatifs depuis le Moyen Âge, comme La maniere de language que t'enseignera bien a droit parler et escrire doulz françois selon l'usage et la coustume de France (XIV ${ }^{e}$ siècle) ou le Vocabulaire pour apprendre à bien lire, escripre et parler françoys et flameng de Noël de Berlaimont paru en 1511 à Anvers chez Willem Vorsterman. (Les Colloquia de Berlaimont incluront progressivement de nouveaux vernaculaires, et iront jusqu'à offrir en 1598 une version octolingue élargie aux diverses langues européennes ${ }^{6}$.) Les langues vulgaires font toutefois progressivement l'objet de travaux lexicographiques. Ainsi le Dictionarium d'Ambrogio Calepino (1502), le fameux Calepin, lexique latin d'abord monolingue, s'enrichit au fil des 
années, augmentant ses entrées latines d'équivalents en vernaculaire, pour accueillir, en 1590, onze langues: latin, hébreu, grec, français, italien, haut-allemand, flamand, espagnol, polonais, hongrois et anglais!

Néanmoins ces outils rudimentaires ne suffisent pas à aider les traducteurs dans leurs tâches. De surcroît, nombreux parmi eux sont ceux qui entreprennent de tourner en français des œuvres écrites dans une langue qu'ils ne maîtrisent pas suffisamment. César Oudin dans l'Avertissement de la seconde édition de ses Proverbes espagnols traduits en françois (1609) raille avec beaucoup d'humour «certains mauvais traducteurs » de l'espagnol, citant en exemple des contresens tirés de traductions contemporaines. Ainsi du «quidam qui a tourné Mona, qui est un singe, ou une guenon en Religieuse, une hache en un flambeau, et plus de cinq cens autres aussi ridicules fautes, en un seul volume» (Oudin 1609: $\mathrm{f}^{\circ} 7 \mathrm{v}^{\circ}$ ). «Vray est,, confesse Oudin, qu'il y a bien des choses difficiles à traduire: mais il faut y apporter du jugement, et ne se point trop fier à ses forces.» Oudin dénonce ainsi la présomption des traducteurs: «[...] la bonne opinion que quelquesfois nous avons de nous mesmes nous fait oublier. Et la metamorphosant en presumption, elle est cause d'autres estranges metamorphoses [...]»(Oudin 1609: fo 7 et $v^{\circ}$ ).

C'est que ces traducteurs "moins experts qu'il ne le seroit de raison» (Oudin 1609: fo 7), selon Oudin, lorsqu'ils sont incertains du sens d'un terme, usent de grécismes, latinismes ou autres calques, parfois jusqu'à l'excès.

S'adressant à ses lecteurs, Geoffroy Tory, au seuil de son célèbre Champfleury (1529), dénonce violemment cette pratique. S'il est attentif à «L'Art et Science de la deue et vraye Proportion des Lettres Attiques», Tory considère que les "écumeurs de latin $»^{7}$, ceux qui corrompent la langue française, sont non seulement dignes du gibet, mais encore qu'il serait bon qu'ils ne fussent jamais nés!

J'estime qu'il y a trois sortes d'hommes qui se plaisent à travailler à la [la langue française] corrompre et à la déformer: ce sont les écumeurs de latin, les faiseurs de bons mots et les jargonneurs. Quand les écumeurs de latin disent: «Despumon la verbocination latiale, et transfreton la Sequane au dilicule et crepuscule, puis deambulon par les Quadrivies et Platees de Lutece, et comme verisimiles amorabundes captiuon la beniuolence de lomnigene \& omniforme sexe feminin", il me semble qu'ils ne se moquent pas seulement de nous, mais d'eux-mêmes. (Tory 1529: fo A8)

Chez Rabelais, l'écolier limousin que croise Pantagruel au chapitre 6 tiendra exactement le même langage! ${ }^{8}$

Tory n'est pas le seul à mener ce combat: les mots « escorchez du Grec ou Latin» sont encore dénigrés par François de Belleforest dans l'Avertissement au lecteur qui précède sa traduction du Second tome des Histoires tragiques de Bandello (1566). Luimême, dit-il, s'efforce d'«enrichir la langue [...] de mots propres». Quant à Henri Estienne, ce sont les italianismes qu'il dénonce dans ses Deux dialogues du nouveau langage français italianisé (1578). Il s'appuie sur l'exemple suivant:

Ce seret une discortesie de passer par la contrade, où est la case des dames que sçavez, s'y faire une petite stanse et toutesfois je ne suis pas maintenant bien acconche pour comparoir devant elles. (Estienne 1578: t. I: 45) ${ }^{9}$

Les expressions idiomatiques présentent des difficultés toutes particulières pour les traducteurs. La première édition bilingue du roman picaresque La Vie de Lazarille de Tormes. Et de ses fortunes et adversitez (Anonyme 1601) pourra en fournir une 
excellente illustration. En effet l'ouvrage, présenté en deux colonnes, comporte de nombreuses expressions calquées sur l'espagnol, mais n'ayant aucun sens en français: «beuvoit plus qu'un donneur de Bonjour / bevia mas que un saludador» (p. 73), «trouver cheute la trampille du Chat / hallar cayda la trampilla del gato» (p. 100), «la pecheresse d'arche / la pecadora del arca» (p. 102), etc. Le traducteur anonyme a cédé à la facilité, se contentant d'aligner des calques, mais laissant le lecteur aux prises avec un non-sens.

Rares sont ceux qui, comme Filbert Bretin, se déclarent parfaits connaisseurs de la langue qu'ils traduisent. Dans son épître "A hauts et puissans seigneurs Guillaume de Saulx [...], Et Jean de Saulx...», il affirme se tenir «tout asseuré de recevoir le choq de plusieurs langues", estimant «qu'il ne se trouvera autre meilleur truchement» (Lucien 1583: fo a ij) que lui pour Les auvres de Lucian de Samosate. Il explique dans le détail la façon dont il a pallié les lacunes lexicales, prenant bien soin de respecter avant tout la langue cible. Selon lui, c'est «le jugement de l'aureille» qui est primordial:

Ayant cependant inventé quelques mots nouveaux, ramené des vieux en usage, et accoursi les noms propres: dont le tout n'a esté par moy fait qu'au bon jugement de l'aureille, qui est la vraye aulne à laquelle on doit mesurer la parolle. S'il se rencontre quelque chose de mon creu de Bourgongne, je n'ay peur qu'on se formalise du transport qui s'en fera hors le pays, veu que toute telle marchandise ne doit rien à la foraine. Des mots de gueulle, s'il y en a, c'est ce que demande Lucian et son ame, avec son parler Comique. (Lucien 1583: fo a ijvo

Conscient des critiques qu'on pourra lui adresser, Bretin revendique la diversité du vocabulaire qu'il emploie, seule à même de rendre le caractère «comique» de son texte source. Provincialismes et «mots de gueulle» - c'est-à-dire «des mots trop libres, paroles déshonnêtes qui se disent parfois dans les repas abondants et joyeux » (Rabelais 1994: 1573 note 4) - sont accueillis dans sa version, au risque de corrompre le «beau langage».

Toutefois dans le domaine de la traduction, l'indécision du vocabulaire, à l'origine de bien des «monstres» lexicaux, comporte aussi des côtés positifs: elle permet d'enrichir la langue de nombreux néologismes, dont certains ont toujours cours aujourd'hui. Hellénismes, latinismes, italianismes abondent dans la chronique gargantuine. Selon Mireille Huchon, «l'apport de Rabelais au vocabulaire français est exceptionnel ${ }^{10} »$.

La néologie n'est cependant pas la seule tactique en cours. Devant l'incertitude lexicale, les traducteurs de la Renaissance recourent parfois à une stratégie promise à un riche avenir: Les Notes du Traducteur. Jean Martin, par exemple, traduisant l'Arcadie (1544), prend soin d'ajouter sous forme de glossaire une «Exposition de plusieurs mots contenus en ce livre, dont l'intelligence n'est commune» (Sannazaro 1544: ff $114 \mathrm{v}^{0}-133 \mathrm{v}^{0}$ ). Il y commente et explicite les noms de lieux et de personnages, classés par ordre alphabétique:

1) [...] Galicio me semble estre un diminutif de Galicinio, ce qui signifie chant du coq. Ou s'il ne l'est, ce poëte veult entendre quelque lien familier Espagnol du pays de Gallice. [...]

2) Lambrusque, c'est vigne sauvage. [...]

3) Selvagio (à mon jugement) est introduit en ces œuvres pour Théocrite. [...] 
4) Thyrsi que Sannazar met pour un pasteur, peult signifier un chapeau nuptial, moyssine, ou lance de Bacchus. [...]

5) Uranio signifiant celeste, est mis en ceste œuvre pour Pontan, qui a faict un œuvre intitulé Urania.

Jean de Maumont, transposant les Histoires et Croniques du Monde de Jean Zonaras (1583) «de leurs nayves langues en vulgaire François», recourt lui aussi à des «annotations mises en la marge pour les diverses lectures grecques et advertissements». Par exemple un commentaire lui semble nécessaire pour le mot «Danois» (p. 3), pour lequel il a trouvé une variante: «En autre livre se lit Damasceniens» (p. 3); de même une correction s'impose pour le mot «seur» (p. 9): «Elle estoit la niece, voyez Suetone et les autres".

De nombreuses autres traductions pourraient encore être mentionnées, comme celle de L'Histoire du monde de Pline déjà citée, au seuil de laquelle Antoine du Pinay place des "Observations recognues après l'Impression", où il commente et justifie ses choix traductifs.

\subsection{Le passage par une langue relais}

La méconnaissance de la langue source est critiquée par de nombreux humanistes. Le plus célèbre en est sans doute Du Bellay qui, dans la Deffense et Illustration de la Langue Françoyse (1549), s'en prend aux «mauvais traducteurs» qu'il compare à des «Reblanchisseurs de murailles» (Du Bellay 2007: 110). Il fustige notamment ceux "qui pour acquérir le Nom de Sçavans, traduysent à credict les Langues, dont jamais ilz n’ont entendu les premiers Elementz, comme l'Hébraique, et la Grecque [...]» (Du Bellay 2007: 89-90).

En effet, nombre de traducteurs ne sont que des «traducteurs de traducteurs». $\mathrm{Au} \mathrm{Xv} \mathrm{x}^{\mathrm{e}}$ siècle déjà, Laurent de Premierfait a traduit «à crédit». Son Livre Decameron autrement surnommé le prince Galleot de Boccace nous est parvenu à travers une traduction latine aujourd'hui perdue d'Antonio d'Arezzo. En témoigne l'explicit: «le quel livre ja pieça compila et escripvi Jehan bocace de certald en langaige Florentin, et qui nagueres a esté translaté Premierement en latin et secondement en françois».

La pratique du recours à une langue-relais perdure toutefois tout au long du $\mathrm{XVI}^{\mathrm{e}}$ siècle. Ainsi, c'est en passant par la traduction latine de Lorenzo Valla que Claude de Seyssel traduit La Guerre du Péloponnèse de Thucydide (1514). D’ailleurs Seyssel fait équipe avec Janus Lascaris, originaire de Constantinople: Seyssel traduit du latin en français, alors que Lascaris traduit du grec en latin. C'est à ce tandem de traducteurs qu'on doit les premières traductions françaises de Xénophon, Appien, Justin ou Diodore de Sicile. Claude de Seyssel s'en explique dans le «Proesme» à sa traduction de l'Anabase de Xénophon qu'il offre en 1504 à Louis XII:

Ainsi je priais ledit Lascaris qu'il voulust ceste histoire me declairer et exposer en latin, afin que je la pusse du latin translater en françois. Lequel l'a tres volontiers fait comme celuy qui de tout son cœur desire faire chose qui vous agree. (Xenophon 1504: $\mathrm{f}^{\circ}$ a ij $\mathrm{r}^{\circ}$ )

On aura la même «traduction de traduction» pour la langue arabe, ou pour l'hébreu, comme en témoigne le Psautier de Marot (1543) ${ }^{11}$. Le phénomène peut se comprendre pour ces langues difficiles, dépourvues d'alphabet latin. Mais il concerne aussi les langues vernaculaires, ce qui peut paraître plus surprenant. Ainsi, c'est par 
l'italien que passe l'espagnol de la Prison d'amour, un roman de Diego de San Pedro, pour être traduit finalement en français en 1525. François Dassy avoue dans son prologue sa dette à l'égard de son ami Lelio de Manfredi qui, à la demande d'Isabelle d'Este, marquise de Mantoue, en avait en 1514 publié une version en italien. On citera encore l'exemple de la traduction anonyme du De claris mulieribus de Boccace, sortie des presses de l'imprimeur lyonnais Guillaume Rouillé en 1551. L'imprimeur s'adresse ainsi «aux lecteurs»:

[...] j'ay bien voulu encore, pour le bien public et proffit d'un chascun, vous mettre en lumiere ce present livre des Dames de renom, premierement faict Latin par iceluy [Boccace], et depuis François par nostre requeste et moyen, suyvant la traduction Italienne du Signeur Luc-Antonio Ridolfi Gentilhomme Florentin. (Boccace 1551 : 3-4)

Henri Estienne, pour sa part, dans la Préface de son Traité préparatif à l'Apologie pour Hérodote (1566), s'en prend violemment à ces «traducteurs des traducteurs»:

Et dont procede ce mal? De ce que ceux qui les ont traduits en ces langues vulgaires, ont esté traducteurs des traducteurs, c'est à dire ont traduict en ces langues les traductions qui en avoyent jà esté faictes en Latin; et n'ayans aucune cognoissance du Grec, non seulement ils ont retenu toutes les fautes de ces traducteurs, mais leur estant avenu souvent de ne les entendre point, sont aussi tombez en plusieurs autres encore plus lourdes et plus vilaines. (Estienne 2007: 103-104)

Selon lui, la mauvaise traduction «défigure» l'original et se montre fautive. Le recours à un langage métaphorique lui permet d'illustrer son propos:

Car je di et maintiens que la plus part des auteurs qui se portent fort bien en Grece, et ont beau visage et bien colouré, sont fort malades, et par consequent sont fort desfaicts voire desfigurez en France, en Italie, en Espagne, et és autres pays, pour le mauvais traitement qu'on leur fait par le chemin. C'est à dire (pour parler clairement et sans allegorie) que plusieurs auteurs, et principalement les Grecs, qui estans lus en leur langage naturel par ceux qui en ont cognoissance suffisante, ont la meilleure grace du monde, et donnent contentement non seulement à l'oreille, mais aussi à l'esprit, sont traduits si pietrement en François, en Italien, en Espagnol, qu'il y a autant de différence de lire leurs livres Grecs ou telles traductions d'iceux, qu'il y aurait de voir le visage d'une mesme personne, quand elle seroit en tresbonne disposition, ou quand apres fort longue maladie elle commenceroit à rendre les derniers souspirs. (Estienne 2007: 103)

Estienne donne l'exemple de Claude de Seyssel, que nous avons cité plus haut, non pas retraduisant Valla, mais plutôt «devinant» ce que Valla voulait dire:

[...] j’ay monstré comment Laurent Valle avoit deviné que vouloit dire Thucydide: et puis le traducteur François, Claude de Seyssel, evesque de Marseille, avoit deviné quavoit voulu dire Laurent Valle: mais comme Laurent Valle avoit mal deviné quelle avoit esté la conception de Thucydide, ainsi Claude de Seyssel avoit esté mauvais devin quant à la conception de Laurent Valle. (2007: 104)

\section{On pourra ainsi faire siennes les conclusions de Romain Menini:}

Traduire du grec à la Renaissance, c'est donc faire doublement «l'épreuve de l'étranger», en passant deux frontières pour le prix d'une. On saisit d'emblée les risques encourus pour arriver jusqu'à la contrée vernaculaire. (Menini 2015: 408)

Il revient à Étienne Dolet d'énoncer la meilleure stratégie pour lutter contre ces incertitudes de la traduction. Son petit traité publié à Lyon en 1540 par ses soins et 
intitulé La Maniere de bien traduire d'une langue en aultre, énonce cinq règles essentielles. La seconde stipule que le traducteur doit parfaitement maîtriser à la fois la langue du texte source et celle dans laquelle il se propose de traduire:

La seconde chose, qui est requise en traduction, c'est, que le traducteur ait parfaicte congnoissance de la langue de l'autheur, qu'il traduict; et soit pareillement excellent en la langue, en laquelle il se mect à traduire. Par ainsi il ne violera, et n'amoindrira la majesté de l'une, et l'aultre langue. [...] Entends, que chascune langue a ses proprietés, translations en diction, locutions, subtilités, et vehemences à elle particulieres. Lesquelles si le traducteur ignore, il faict tort à l'autheur, qu'il traduict: et aussi à la langue, en laquelle il le tourne: car il ne represente, et n'exprime la dignité, et richesse de ces deux langues, desquelles il prend le manîment. (Dolet 1540: ff b2v-b3)

\subsection{Domaine culturel et politique}

Mais l'incertitude de la traduction provient parfois aussi de difficultés d'ordre interculturel. Il est difficile en effet pour les hommes de la Renaissance de renoncer à l'ethnocentrisme. La traduction du roman espagnol Question de amor intitulée le Debat des deux gentilzhommes espaignolz (1541) l'atteste. Le traducteur anonyme n'est pas familier des jeux et divertissements de la cour hispano-napolitaine et renonce par conséquent à traduire la description qui en est donnée: «Le jeu des eaues n’a aulcun propre terme Françoys à tout le moins que le sache pour n'estre point en usage» (1541: f. $\left.45 \mathrm{r}^{\circ}\right)$. De plus, la symbolique des couleurs lui échappe: "Car aussi le blason des couleurs est aultre en Espaigne que au pays de France» (Anonyme 1541 : f. $45 \mathrm{r}^{\circ}$ ). De même, il refuse de traduire la longue egloga (églogue rendue par «bergerie»), forme poétique qu'il ne maîtrise pas et qu'il dénigre: «Mais passons outre et remettons ces querelles et exclamations tragedieuses à la cerymonie des Espaignolz» (1541: f. $45 \mathrm{r}^{\mathrm{o}}$ ).

Une dimension politique vient de surcroît compliquer la traduction et ajouter à l'incertitude ${ }^{12}$. La première moitié du XVI $\mathrm{X}^{\mathrm{e}}$ siècle accuse ainsi la rivalité entre la France et l'Espagne. Dans le Debat des deux gentilzhommes espaignolz, l'évocation de la «cruelle bataille de Ravenne» (11 avril 1512), qui clôt le récit, fait par exemple l'objet d'adaptations importantes sous la plume du traducteur. Ce dernier supprime la description du défilé de l'armée du vice-roi avant son départ de Naples ainsi que le songe prémonitoire du héros Vasquiran, qui dans le texte espagnol présentait les combattants espagnols comme des martyrs de la foi. À la place, il insère un éloge de l'armée française et de son commandant, Gaston de Foix-Nemours, «dompteur fatal des adversaires de France» (1541: $\left.\mathrm{f}^{0} 67 \mathrm{v}^{\circ}\right)$. Il est vrai que les Français l'emportèrent sous la bannière de ce chef et « recordz de leur ancienne prouesse», «remirent en memoire aux Espagnolz et Italiens, le victorieulx exercite de Boemus» (1541: fo $\left.67 \mathrm{v}^{0}\right)$. Mais ce fut une victoire à la Pyrrhus, le jeune général y perdant la vie (parmi des milliers de morts), et l'armée étant obligée de rebrousser chemin, laissant finalement le champ libre aux Espagnols. La traduction s'envisage donc ici comme une (re)conquête symbolique des nations rivales.

On signalera de plus un brouillage d'origine commerciale et publicitaire, avec des textes faussement présentés comme des traductions. Par exemple, il semble aller de soi qu'un livre retraçant les aventures d'Amadis de Gaule et de ses descendants soit traduit de l'espagnol - fût-il en réalité traduit de l'italien (Les Quinzième, Seizième, Dix-septième, Dix-huitième, Dix-neuvième, Vingtième et Vingt et unième 
Livres d'Amadis sont empruntés au Sferamundi di Grecia de Mambrino Roseo ${ }^{13}$ ) ou même de l'allemand (Les Vingt et Deuxième, Vingt et Troisième, Vingt Quatrième et dernier livre $\left.{ }^{14}\right)$. À l'inverse, il arrive que la traduction ne soit pas avouée. C'est le cas de la Penitence d'amour, traduite par René Berthault de la Grise (1537) et qui rassemble des passages extraits de plusieurs romans sentimentaux espagnols (Penitencia de amor, Question de amor, Grimalte y Gradissa... $)^{15}$. L'incertitude règne donc sur le texte lui-même: s'agit-il, ou non, d'une traduction? La réponse n'est pas toujours aisée... De surcroît, l'attitude désinvolte du traducteur renaissant s'ajoute souvent à l'incertitude. Nombreuses sont les préfaces de traductions qui évoquent cette liberté du traducteur, en la revendiquant ou au contraire en la dénonçant. Nous en donnerons ici trois exemples ${ }^{16}$.

Tout d'abord François Dassy et son Dialogue treselegant intitule le Peregrin traictant de l'honneste et pudicq amour concilie par pure et sincere vertu [...], traduit de Giacomo Caviceo $(1531)^{17}$. Dans son «Proesme de l'acteur», Dassy s'adresse ainsi à une dame anonyme:

Bien certain suis que aucuns me vouldront imputer n'avoir ensuy [sic] de point en point l'Italique translation. Ce que j'ay faict pour esclarcir aucunes scabreuses et difficiles sentences en acomplissant ce qui vous a pleu de grace me commander. (Caviceo 1531: $\left.f^{\circ} 6 v^{0}\right)$

C'est dans un souci de clarification du sens que le traducteur déclare s'écarter de son original.

La liberté du traducteur est encore revendiquée par Jacques Mondot dans l'avertissement «Au Lecteur» en tête de sa traduction des Cinq livres des Odes de Q. Horace Flacce (1579) :

Je ne doute poinct que tu ne die qu'en plusieurs endroicts de ceste traduction je suys plus tost ma fantasie, que la diction de mon autheur, et que mes vers sont rudes, contraints, et malgracieux, à quoy je te respons, que licet non iurarim in verba magistri, tu ne me dois mettre le fardeau sur les espaules, mais plustost t'en prendre ou à la difficulté et briefveté d'Horace ayant emprunté plusieurs façons de parler de Pindare, Anacreon et autres poetes Grecs, ou bien à la liberté de nostre langue Françoise, laquelle estant estranglee et contrainte perd ou du tout ou grande partie de sa grace. J'ay toutesfois visé au but de son intention au plus pres qu'il m’a esté possible. (Horace 1579: fo e iij vo

Reprenant une formule d'Horace ("Nullius addictus jurare in verba magistri», «sans être contraint de jurer sur les paroles d'aucun maître», Épîtres I, 1, v. 14), Mondot affirme avec fierté n'être inféodé à aucune école, fût-elle celle de l'auteur lui-même qu'il traduit! Conscient d'être un pionnier ("c'est chose bien difficile de ne s'esgarer en un chemin par où personne n'a encore passé»), il rejette la responsabilité de ses éventuelles «faute[s] legere[s] » sur l'imprimeur, et attribue le caractère «fantaisiste» de sa traduction à «la difficulté et briefveté d'Horace» ainsi qu'à «la liberté de nostre langue Françoise».

C'est donc parfois au nom de ce qu’on a pu appeler le «génie de la langue » qu'est revendiquée la liberté du traducteur, comme le signale encore Jean de Maumont dans l'Avis «Aux Lecteurs» qui précède sa traduction du Premier livre des Histoires et Chroniques du monde de Jean Zonaras: «nostre langue Françoise, [...] a ses phrases et manieres de parler separees de l'Hebrieu, Grec, et Latin» (Zonaras 1583: f a ij vo). 
Le texte doit alors avant tout plaire aux oreilles du lecteur français, comme l'indique la «cinquième reigle que doibt observer ung bon traducteur», selon Dolet, qui prône:

L'observation des nombres oratoires: c'est asscavoir une liaison, et assemblement des dictions avec telle doulceur, que non seulement l'ame s'en contente, mais aussi les oreilles en sont toutes ravies, et ne se faschent jamais d'une telle harmonie de langage. (Dolet 1540: ff b4)

\subsection{Jean de Maumont}

Ce même avertissement "Aux Lecteurs» offrira pour conclure une excellente synthèse au sujet de l'incertitude de la traduction au $\mathrm{xvI}^{\mathrm{e}}$ siècle. En effet, Jean de Maumont y évoque toutes les difficultés qu'il a rencontrées lors de son travail, et décrit les stratégies qu'il a adoptées. Nous en dégagerons trois principales.

Maumont rappelle que l'incertitude gouverne le genre même de la traduction, si proche de l'imitation, de la paraphrase, ou de l'adaptation. Sans crainte d'être "reprins ou blasmé», Maumont «dilat[e] les clauses par maniere de paraphrase» et "amplifie» son texte source, puisant chez d'autres auteurs, dans le but, dit-il, d'«esclarcir le poinct de l'Histoire»:

Si donques en tels endroits et lieus j'ay paraphrasé parfois le texte, et supply pour son remplissage, les clauses d'iceluy, en y amenant les lieus entiers desdits aucteurs Hebrieus et Grecs, je n'en doy estre reprins ne blasmé en ma translation et racueil, veu que l'ay fait pour la commodité de ceux, qui n'auroient le Grec ou l'Hebrieu en main, pour visiter chaque coup les passages, et esclarcir le poinct de l'Histoire: et aussi pour donner quelquefois grace à l'oraison, le moins mal que j'ay peu, sans rien laisser ce pendant de l'Aucteur, ny ajoindre chose, qui offense, ou altere son intention, sens et vouloir, et qui ne face cours et declaration de l'Histoire, qu'il traitte, ne qui ne soit prinse de mesme lieu et ancien livre Grec, dont il a pestri et massonné toutes les circonscriptions et traits de son recit et discours. Et toutes-fois là mesme, ou j'ay tracé et amplifié le papier, non de moy mesme, mais prins du fons et champ desdits volumes Hebrieus et Grecs, je l'ay ou coté sur la marge, ou marqué (pour satisfaire aux gens doctes en la langue, qui peut estre, voudront conferer quelques lieus [...]). (Zonaras 1583: $\mathrm{f}^{\circ}$ a ij v ${ }^{\circ}$ )

Sa justification est toutefois double, puisqu'à une justification pratique et pédagogique ("commodité») s'ajoute une justification esthétique et stylistique ("pour donner quelquefois grace à l'oraison»). Maumont insiste tout du long sur le caractère intègre de son travail: non seulement il a respecté «l'intention» de l'auteur, mais en plus il a pris soin de signaler scrupuleusement tout ajout par emprunt.

Pour supprimer les incertitudes sur le plan textuel, Maumont entreprend un double travail lexical et structurel:

[...] je l' [ce present tome] ay mis et divisé en certains livres separez l'un de l'autre, et imprimez chacun à part, Annexez et conjoincts en un volume pour l'aisance et plaisir du lecteur, pour lequel respect aussi ay comparty et façonné chaque livre par distinctions et pauses d'Histoires et récits, scachant bien que lire escrits de si longue haleine, est chose ennuyeuse, et que l'intermission et repos plaist et recree. [...] Aussi quant aux forteresses, instrumens de baterie, description de lieus, batailles donnees, j'ay parfois usé en telles matieres des vocables et termes qui sont aujourdhuy en vigueur, és choses toutes-fois, qui desja lors estoient en usage: mais plusieurs d'elles n'avoient encor receu tels noms, aptes et propres à leur forme et nature [...]. (Zonaras 1583: $\mathrm{f}^{\circ}$ a ij vo) 
Maumont introduit donc des divisions dans le texte pour en faciliter la lecture. De même, il modernise ou adapte le vocabulaire de façon à être mieux compris de son lecteur. Ainsi les « ouvrages de guerre» («comme du temps des premiers Hebrieus les vineas, ou vignes, belins ou moutons, palevesates, parapects, cavaliers mantelets, gabions, enseignes») n'étaient pas encore «cognus ny nommez à la mode, que les Romains les appelerent apres, ny du temps aussi des premieres Monarchies des Assiriens et Perses", constate-t-il;

[...] mais ils avoient autres engins de force et defense, qui neantmoins estoient, ou de semblable forme, ou d'effet egal à ceux là, si bien le nom n'en estoit lors tel: et pour les donner à entendre, il m'a falu les exprimer quelquefois par les noms, qui furent apres imposez à semblables choses. (Zonaras 1583: $\mathrm{f}^{\circ}$ a ij v ${ }^{\mathrm{o}}-$ iij $\mathrm{r}^{\circ}$ )

Le traducteur affirme agir ainsi pour la «cognoissance et plaisir» de son lecteur.

Enfin Maumont résout les incertitudes concernant la responsabilité morale et juridique des opinions exprimées dans le texte.

[Le «translateur»...] soumet sa translation telle qu'elle est, au jugement des gens de bien et des sçavans, qui jugent sans malveillance et perverse affection. Et quant aux particulieres opinions, si aucunes y en a [...] qui ne sont siennes, ains de l'aucteur Grec, ou de ceux, dont Zonoras les a tirees: car ledit Aucteur en tout et par tout fait office de recueilleur et recitateur du dire d'autruy, se remetant de ce qui n'en est, sur la foy de celuy, dont il l'a tiré sans prendre rien sur sa charge: le translateur en sa traduction aussi, soumet toutes ces choses au consentement, et censure des saintes Academies et doctes escholes de ce Royaume [...]. (Zonaras 1583: $\mathrm{f}^{\circ}$ a iij $\mathrm{r}^{\circ}$ )

Rapprochant subtilement la démarche du traducteur de celle de l'auteur-compilateur («recueilleur et recitateur du dire d'autruy») et soumettant le texte ainsi rassemblé «sans prendre rien sur sa charge», Maumont se décharge de toute responsabilité. Il prend toutefois soin d'adresser son travail aux lecteurs bénévoles, ces "gens de bien et [...] sçavans, qui jugent sans malveillance et perverse affection». Soit le lecteur idéal de tout traducteur aujourd'hui encore.

\section{Conclusion}

$\mathrm{Au} \mathrm{XVI} \mathrm{I}^{\mathrm{e}}$ siècle, alors que se multiplient en France les traductions, et qu'apparaissent les premières réflexions théoriques systématiques sur la traduction, l'entreprise traductive n'est guère aisée. Qu'il s'agisse de répondre à une commande royale, visant à diffuser largement les savoirs, ou de satisfaire à la demande des imprimeurs, soucieux d'alimenter leurs presses, les traducteurs se trouvent souvent devant l'incertitude. Si l'article 110 de l'Ordonnance de Villers-Cotterets (1539) exige que les actes juridiques «soient faits et écrits si clairement, qu'il n'y ait ni puisse avoir aucune ambiguïté ou incertitude ni lieu à demander interprétation ${ }^{18}$ », tant s'en faut que les textes auxquels se consacrent les traducteurs soient faciles à tourner en français. Aux difficultés matérielles s'ajoutent les obstacles linguistiques, culturels ou politiques.

Cependant les stratégies mises en place par les hommes de la Renaissance pour contrer ces incertitudes sont pour certaines toujours d'actualité. Ainsi de l'art de la collation, qui vise à restituer les leçons optimales d'un texte original parfois corrompu. Ou encore de l'usage des notes du traducteur, qui exposent et justifient des partis pris de traduction. La mise en place progressive d'une éthique, voire d'une 
déontologie de la traduction, se traduit aujourd'hui dans le décentrement de la position du traducteur, qui se prête plus volontiers à l'interculturel. Enfin les outils de la traduction qui ont timidement apparu au cours du $\mathrm{xvI}^{\mathrm{e}}$ siècle - grammaires, lexiques et autres dictionnaires - se voient perfectionnés chaque jour davantage à l'ère informatique.

Si la frénésie de traduction est toujours de mise au $\mathrm{xxI}^{\mathrm{e}}$ siècle, l'accroissement massif du volume des traductions à la Renaissance étant comparable, selon Berman, «à celui qui a eu lieu dans la seconde moitié du $\mathrm{xx}^{\mathrm{e}}$ siècle» (1988: 23), il convient toutefois de ne pas condamner à l'obsolescence la Maniere de bien traduire d'une langue en aultre de Dolet - n'importe qui pouvant de nos jours encore s'improviser traducteur, même et surtout les machines, au risque de l'incertitude.

\section{NOTES}

1. Voir le corpus en Annexe.

2. Voir, par exemple: Olivier-Bonfils, Delphine (2013): Compte rendu de Berman, Antoine (2012): Jacques Amyot, traducteur français. Essai sur les origines de la traduction en France. Paris: Belin. Meta. 58(3):670-672.

3. Cette traduction sera republiée quatre fois: Complainte tres piteuse de Flammette a son amy Pamphile. Translatee Ditalien en vulgaire Françoys. Paris: J. Longis (1532); Complainte tres piteuse [...]. Lyon: François Juste (1532); Complainte des tristes amours de Flammette a son amy Pamphile. Lyon: Claude Nourry (1532); Complainte tres piteuse [...]. Paris: Denis Janot (1541).

4. Boccace (2003) Fiammetta. Traduit de l'italien par Serge Stolf. Paris: Arléa.

5. Jean Oporin avait fait paraître l'année précédente l'editio princeps grecque des Oracles sibyllins, préparée par Xystus Betuleius.

6. De Berlaimont, Noël (1598): Colloquia et dictionarum octo linguarum. Delft: Schnickel. Le lexique de Berlaimont restera en vogue jusqu'au milieu du xvirI ${ }^{\mathrm{e}}$ siècle.

7. Les «Forgeurs de mots nouveaulx» ne reçoivent pas un meilleur accueil: «Si telz Forgeurs ne sont Ruffiens/ je ne les estimes gueres meilleurs. Pensez qu'ilz ont une grande grace / quant ils disent apres boyre, quiz ont le Cerveau tout encornimatibule / et emburelicoque dung tas de mirilifiques et triquedondaines, dung tas de gringuenauldes, et guylleroches qui les fatrouillent incessamment?» (Tory 1529: fo A8 vo).

8. «[...] nous despumons la verbocinatiun Latiale et comme verisimiles amorabonds captons la benevolence de l'omnijuge omniforme et omnigene sexe feminine [...]. (Rabelais 1994: 233).

9. La critique toutefois s'accorde pour estimer qu'Estienne exagère, les termes en italiques n'étant attestés que chez cet auteur. Seul acconche, de l'ital. acconcio, "commode, accommodé», à côté de conche, «ajustement» (ital. concio), a eu une existence passagère.

10. Rabelais (1994) Notice sur la langue de Rabelais, p. XLVI. M. Huchon cite de nombreux exemples, parmi lesquels les hellénismes: acromion, encyclopédie (Pantagruel), automate, hippiatrie (Gargantua); les latinismes: célèbre, indigène (Pantagruel), athlétique, factice (Gargantua); les italianismes: gondolier, torticolis (Pantagruel), $\mathrm{c}[\mathrm{h}]$ aresse, carrière (Gargantua), etc.

11. Marot a également traduit du grec «à credict» Héro et Léandre de Museus, à partir de la traduction latine de Guillaume des Mores.

12. Sur la dimension conquérante de la traduction, qui devient le "substitut " d'une conquête politique et militaire, on pourra lire les travaux de Jean Balsamo, notamment (1992): Les Rencontres des muses (Italianisme et anti-italianisme dans les Lettres françaises de la fin du XVI siècle). Genève: Slatkine.

13. Quinzième Livre d'Amadis, traduction G. Chappuys (1577) et A. Tiron (1577); Seizième Livre d'Amadis, traduction N. de Montreux (1577) et G. Chappuys (1578); Dix-septième Livre d'Amadis, traduction G. Chappuys (1578); Dix-huitième Livre d'Amadis, traduction G. Chappuys (1579); Dix-neuvième Livre d'Amadis, traduction J. Charlot (1581) et G. Chappuys (1582); Vingtième Livre d'Amadis, traduction G. Chappuys (1581) et J. Boyron (1582); Vingt Unième et Dernier Livre d'Amadis, traduction G. Chappuys (1581).

14. Vingt et Deuxième Livre d'Amadis (1615), Vingt et Troisième Livre d'Amadis (1615), Vingt Quatrième et dernier Livre d'Amadis (1615). 
15. Berthault de la Grise, René (1537): La penitence d'amour. Lyon: Denis de Harsy. Voir à ce sujet Duché, Véronique (2008): Si du mont Pyrenée / N'eussent passé le haut fais.... Les romans sentimentaux traduits de l'espagnol en France au XVI siècle. Paris: Champion, notamment les pages 406 à 413.

16. Pour une étude plus ample, voir Duché, Véronique, dir. (2015) et tout particulièrement le Chapitre II: «La traduction vue par les traducteurs» de Sebastián García BARRERA et Pascale Mounier. 127-182.

17. Voir Caviceo, Giacomo (1531). Il s'agit de la deuxième édition, le texte étant «reveu au long et corrigé oultre la premiere impression avec les annotations et cottes sur chascun chaspitre par Jehan Martin».

18. Ordonnance $\mathrm{n}^{\circ} 188$ de 1539 du roi François I ${ }^{\text {er }}$. Villers-Cotterets.

\section{RÉFÉRENCES}

Adam, Renaud et VAnautgaerden, Alexandre (2009): Thierry Martens et la figure de l'imprimeur humaniste: une nouvelle biographie. Coll. "Nugæ humanisticaæ sub signo Erasmi». 11(1). Turnout: Brepols.

Belle, Marie-Alice, dir. (2012): Women's Translations in Early Modern England and France/La traduction au féminin en France et en Angleterre (XVI et XVII siècles). Numéro spécial. Renaissance and Reformation/Renaissance et Réforme. 35(4):5-23.

BERMAn, Antoine (1988): De la translation à la traduction. TTR. 1(1):23-40.

Berman, Antoine (2012): Jacques Amyot, traducteur français. Essai sur les origines de la traduction en France. Texte établi par Isabelle Berman et Valentina Sommella. Coll..«L'Extrême Contemporain ». Paris: Belin.

Chavy, Paul (1988) Traducteurs d'autrefois. Moyen Âge et Renaissance: Dictionnaire des traducteurs et de la littérature traduite en ancien et moyen français (842-1600). Genève: Slatkine.

Delisle, Jean et Woodworth, Judith, dir. (1995): Les traducteurs dans l'histoire. Ottawa: Les Presses de l'Université d'Ottawa.

Delisle, Jean, dir. (2002): Portraits de traductrices. Ottawa/Arras: Les Presses de l'Université d'Ottawa/Artois Presses Université.

Duché, Véronique, dir. (2015): Histoire des Traductions en Langue Française, XVet XVI siècles. Paris: Verdier.

Furno, Martine (1999-2000) : Les dictionnaires de Robert Estienne: Sens et finalités d'une œuvre lexicographique. Voces. 10-11:11-27.

Hauvette, Henri (1909): Les plus anciennes traductions françaises de Boccace (XIVe-XVII siècle). Bordeaux: Feret et fils.

Huchon, Mireille (2007): Traduction, translation, exaltation et transmutation dans les Amadis. Cameno. 3.

Menini, Romain et Worth-Stylianou, Valérie (2015): "Langues anciennes». In: Véronique Duché, dir. Histoire des traductions en langue française, $X V^{e}$ et XVI siècles. Paris: Verdier, 383-425

Pym, Anthony (1997): Pour une éthique du traducteur. Ottawa/Arras: Les Presses de l'Université d'Ottawa/Artois Presses Université.

Renouard, Philippe (1969): Imprimeurs et libraires parisiens du XVI siècle. Volume 2. BaaleuBanville. Coll. «Histoire générale de Paris». Paris: Service des travaux historiques de la Ville de Paris.

Roessli, Jean-Michel (2013) : Sébastien Castellion et les Oracula sibyllina: Enjeux philologiques et théologiques. In: Marie-Christine Gomez-Géraud, dir. Sébastien Castellion. Des Écritures à l'écriture. Paris: Classiques Garnier, 223-238.

Stolf, Serge (2008): Traductions et adaptations françaises de l'Elegia di madonna Fiammetta. Cahiers d'études italiennes. 8:177-194.

VAn Hoof, Henri (1991): Histoire de la traduction en Occident, Paris/Louvain-la-Neuve: Duculot. Worth, Valerie (1988): Practising Translation in Renaissance France: The Example of Étienne Dolet. Oxford: Clarendon Press. 


\section{ANNEXE}

\section{Corpus}

Achille StATIUs (1575): Les amours de Clitophon et de Leucippe, escrits jadis en grec par Achilles Statius Alexandrin, et depuis mis en latin par L. Annibal Italien, et nouvellement traduits en langage françois par B. Comingeois. Traduit du latin par François de Belleforest. Paris: Jean Borel.

Anonyme (1541): Le debat des deux gentilzhommes espagnolz sur le faict d'amour. Traduction anonyme de l'espagnol. Paris: Denis Janot.

Anonyme (1546): Oracula Sibylina. Traduit du grec ancien par Sébastien Castellion. Bâle: Jean Oporin.

Anonyme (1601): La Vida de Lazarillo de Tormes. Y de sus fortunas y adversidades. La Vie de Lazarille de Tormes, Et de ses fortunes et adversitez. Traduction nouvelle, Raportée et conferée avec l'espagnol, par M.P.B. Parisien. Traduit de l'espagnol par Pierre Bonfons (?). Paris: Nicolas et Pierre Bonfons.

BANDELlo, Matteo (1566): Le second tome des Histoires tragiques, extraites de l'italien de Bandel, contenant encore dixhuit histoires traduites et enrichies outre l'invention de l'autheur par François de Belleforest. Traduit de l'italien par François de Belleforest. Paris: R. Le Mangnier.

Bible (1535): La Bible. Qui est toute la Saincte Escripture. Traduit du grec ancien par PierreRobert Olivétan. Neuchâtel: Pierre de Vingle.

Boccace (1401-1500): Livre appellé Decameron, autrement surnommé le Prince Galiot. Traduit du latin par Laurent de Premierfait. Manuscrit.

Boccace (1551): Des Dames de renom. Traduction anonyme parfois attribuée à Denis Sauvage. Lyon: Guillaume Rouillé.

BocCACE (1585): La Fiammette amoureuse de M. Iean Boccace gentil-homme florentin: contenant, d'une invention gentile, toutes les plainctes et passions d'amour. Faicte Françoise et Italienne, pour l'utilité de ceux qui desirent apprendre les deux langues, par G.C.D.T. Traduit de l'italien par Gabriel Chappurs. Paris: Abel L’Angelier.

Calepino, Ambrogio (1502): Dictionarium. Reggio Emilia: Dionysius Bertochus.

Calepino, Ambrogio (1590): Dictionarium. Bâle: Sébastien Henricpetri.

Caviceo, Giacomo (1531): Dialogue treselegant intitule le Peregrin traictant de l'honneste et pudicq amour concilie par pure et sincere vertu [...]. Traduit de l'italien par François DAssy et révisé par Jean Martin. Paris: Antoine Couteau, Alain Lotrian.

Dolet, Étienne (1540): La Manière de bien traduire d'une langue en aultre. Lyon: Étienne Dolet.

Du Bellay, Joachim (2007): Deffense et Illustration de la Langue Françoyse. Jean-Charles Monferran et Ernesta Caldarini, dir. Genève: Droz.

EsTIENNE, Henri (2007): Introduction au traité de la conformité des merveilles anciennes avec les modernes ou Traité préparatif à l'Apologie pour Hérodote [1566]. Éd. B. Boudou. Genève: Droz.

Estienne, Henri (1578): Deux dialogues du nouveau langage françois italianizé, et autrement déguizé, principalement entre les courtisans de ce temps. Genève.

HÉLIODORE (1547): L'Histoire aethiopique de Heliodorus, contenant dix livres, traitant des loyales et pudiques amours de Théagènes Thessalien et Chariclea Aethiopienne, nouvellement traduite de grec en françoys. Traduit du grec ancien par Jacques Амчот. Paris: J. Longis.

Héliodore (1559): L'Histoire Aethiopique de Heliodorus, contenant dix livres, traitant des loyales et pudiques amours de Theagenes Thessalien et Chariclea Aethiopienne. Traduite de Grec en François, et de nouveau reveüe et corrigée, sur un ancien exemplaire escript à la main, par le translateur, ou est déclaré au vray qui en a esté le premier auteur. Traduit du grec ancien par Jacques Амүот. Paris: J. Longis et R. Le Mangnier.

Horace (1579): Les cinq livres des Odes de Q. Horace Flacce, traduits du latin en vers françois par J. Mondot Velaunois. Traduit du latin par Jacques Mondot. Paris: Nicolas Poncelet.

Justin (1559): Euvres de sainct Justin Philosophe et martyr, mises de Grec en langage François. Traduit du grec ancien par Jean DE MaUmont. Paris: Vascosan. 
LuCien (1583): Les auvres de Lucian de Samosate. Traduit du grec ancien par Filbert Bretin. Paris: A. L'Angelier.

Marot, Clément (1543): Cinquante pseaumes en francois par Clem. Marot. Genève: Jean Gérard. Mexía, Pedro (1526): Les diverses leçons de Pierre Messie Gentil-homme de Sevile. Mises de castillan en françois par Claude Gruget, Parisien. Avec sept dialogues de l'autheur, dont les quatre derniers ont esté de nouveau traduits en cette quatriesme edition [...]. Reveu de nouveau en ceste derniere Edition. Traduit de l'espagnol par Claude Gruget. Lyon: Claude Michel

Oudin, César (1605/1609): Refranes o Proverbios traduzidos en lengua Francesa. Proverbes espagnols traduits en François. Paris: M. Orry.

Pline (1562) L'Histoire du monde de C. Pline Second. Traduit du latin par Antoine Du Pinet. Lyon: Claude Senneton.

Rabelais, François (1994): Euvres complètes. Mireille Huchon, dir. Coll. «Bibliothèque de la Pléiade». Paris: Gallimard.

Sannazaro, Jacopo (1544): L'arcadie de messire Jacques Sannazar, gentilhomme Napolitain, excellent poete entre les modernes, mise d'Italien en Françoys par Jehan Martin [...]. Traduit de l'italien par Jean Martin. Paris: M. Vascosan et G. Corrozet.

San Pedro, Diego de (1525): Prison d'amour. Traduit de l'espagnol par François Dassy. Paris: Galliot du Pré.

Tory, Geoffroy (1529): Champfleury: Auquel est contenu L'art et Science de la deue et vraye Proportion des Lettres Attiques, qu'on dit autrement Lettres Antiques, et vulgairement Lettres Romaines proportionnees selon le Corps et Visage humain. Paris: Geoffroy Tory et Gilles Gourmont.

Xenophon (1504): Anabase. Traduit du grec ancien par l'intermédiaire du latin par Claude DE SeYsSEL. BnF ms fr. 701.

Xenophon (1529): Histoire du voyage que fit Cyrus a lencontre du Roy de Perse Artaxercès son frere par messire Claude de Seyssel, jadis evesque de Marseille. Traduit du grec ancien par Claude De SeYssel. Paris: Galliot Du Pré.

Ximénez de Urrea, Pedro Manuel (1537): Penitence d'amour. Traduit de l'espagnol par René Berthault de la Grise. Lyon: Denis de Harsy.

Zonaras, Jean (1583): Les Histoires et Croniques du monde de Jean Zonaras [...] disposez en trois livres. Le Premier fait mention de l'estat des choses passées en Judée, Perse, Égypte et Grèce... Mis de nouveau de leur nayves langues en vulgaire françois, par J. de Maumont [...]. Traduit du grec en françois, avec annotations mises en la marge pour les diverses lectures grecques et advertissements et tables [...]. Traduit du grec ancien par Jean DE MAUmont et Jean Millet de Saint Amour. Paris: J. Houzé. 